\title{
Robert R. Ruffolo, Jr
}

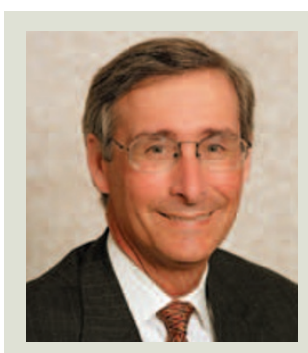

Robert R. Ruffolo, Jr, President, Research and Development, and Corporate Vice President, Wyeth Pharmaceuticals

Robert R. Ruffolo joined Wyeth in 2000 as Executive Vice President, responsible for Pharmaceutical Research and Development. Before this he had a 17-year tenure at SmithKline Beecham Pharmaceuticals as Senior Vice President and Director of Biological Sciences, Worldwide, and spent 6 years at Lilly Research Laboratories. He is a pharmacist and pharmacologist by training, having obtained his B.S. and Ph.D. degrees from the University of Ohio, followed by a postdoctoral fellowship at the National Institutes of Health. Ruffolo has been key to the discovery of several blockbuster drugs, and has been particularly recognized for his pioneering research on carvedilol (Coreg/Kredex), the current standard of care treatment for heart failure. In 2004, the American Chemical Society recognized Ruffolo for 'Leadership in creating a research environment that promoted innovation', and awarded him its Heroes of Chemistry Award.

You famously set metrics for the quantity of compounds you wanted to see move through Wyeth's pipeline. How did you ensure that quality wasn't compromised?

When we set up our metrics-based system, we had to redefine the quality that every single compound had to achieve before it moved into development. Project leaders won't make recommendations to put a drug in to development unless it meets about 20 different criteria including potency, innovation, drug metabolism, safety and dosing frequency. The compound is also considered by a product development council comprising different stakeholders who vote on whether to move it to the next phase, so the metrics system can't be gamed by putting in compounds of lower quality. In fact the exact opposite happened - instead of these groups battling one another, they started to cooperate and quality actually increased.

\section{Is there any evidence that this approach is working?}

When we started, the industry success rate for getting a compound into Phase I, according to the Centre for Medicines Research (CMR), was $70 \%$, and it has since fallen to $55 \%$.

Wyeth's success rate for Phase I has gone up to $80 \%$ and we're equal to or better than the industry standard for getting compounds into Phase II and Phase III. Further downstream, our objectives were to submit two New Drug Applications (NDAs) per year, every year. Last year we hit that target, and we'll have four this year and at least two NDAs in 2007. Before we introduced the metrics, we were only putting three drugs into development per year. There's no way you're going to turn three drugs going into Phase 0 into two NDAs.

\section{Are you now setting new metrics in other parts} of the process?

Yes. With the new Early Clinical Development Centers (ECDCs) we're now setting targets for enrolment. For example, we know how many patients we have to screen to get the required level of enrolment and so we will monitor that, and if we're screening very high numbers and get only few patients then we know that our screening criteria are wrong.

\section{What is the status of the ECDCs?}

We currently have 10 ECDCs up and running and we plan to expand that by at least another five by the end of 2006. We're moving as much as possible away from the US and Western Europe. We're not leaving those locations entirely — the ECDCs will probably do $80 \%$ of our trials - but the availability of patients is far greater in regions such as Eastern Europe, Asia-Pacific and Latin America. Moreover, clinical trial performance statistics, again according to CMR, show that US and European sites are not performing very well. Most of the ECDCs are being set up to run all types of clinical trial, but there might be limitations, such as when a particular disease does not occur in a specific country. We're also treating cancer a little bit differently too, in that we will have ECDCs that will be devoted primarily, but not exclusively, to cancer.

How much time will the ECDCs save?

We set out to save time, but it turns out we'll save costs too. When I joined Wyeth it took
18 months to do a Phase II trial - it now takes 3 years. So, at the very least we want to cut 18 months off the length of Phase II trials, maybe more. We will save costs for two reasons: first, the cost of treating patients in other countries is lower, and second, it's a far more efficient system. Right now when you do a Phase II clinical trial you might do it in 100-200 sites around the world, and for all of those sites you've got to monitor the trials, send and recover the drug from all sites and audit all those sites. If, instead, we're doing most of our Phase II studies in 5-15 sites around the world, you can imagine the cost savings.

\section{How will you account for different pre- treatment history and loss of population heterogeneity?}

Although this was never our intention, it happens to be the case that most of the patients in the regions we're moving to haven't been previously treated with anything, either because there are no treatment options, or fewer options than in the US and Europe, for that particular disease. Population heterogeneity is a potential problem, but we will never do all our studies at a single ECDC and so will have a cross-section of people from various regions. Genetic variations, although they do occur, rarely make a therapeutic difference, and we're willing to take that chance because we think the gains in efficiency will more than offset the rare event where we have to terminate development because we get a drug effect that is unique to a single population.

\section{Will the ECDCs make it easier to adopt novel trial designs?}

We have been working closely with regulators on adaptive trial designs. I think they are quite comfortable with adaptive trial designs in Phase II, but are less receptive right now to the use of adaptive trial designs in Phase III. However, we're seeing signs that they might be open to further discussion, although this is an area in which the European regulators might be more progressive in their thinking than the FDA. Of course the regulators never move as rapidly as wed like them to, but I'm encouraged by some of the more recent progress that I've seen.

Interview by Joanna Owens 